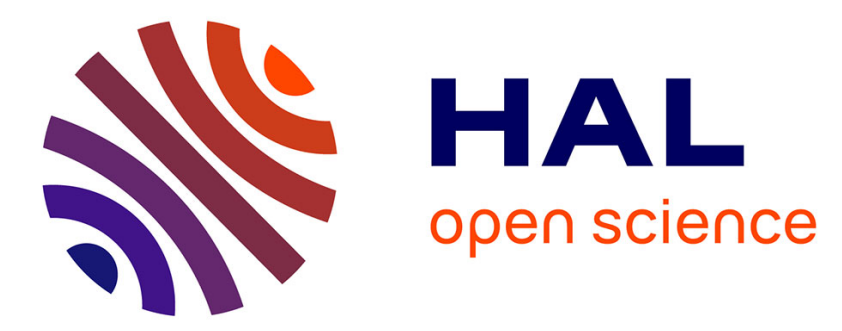

\title{
Structure de la variabilité du rendement du blé d'hiver dans quelques départements céréaliers français. I. - Variabilité entre années et entre départements du peuplement épi et du poids de grain par épi \\ Martine O. Guerif
}

\section{To cite this version:}

Martine O. Guerif. Structure de la variabilité du rendement du blé d'hiver dans quelques départements céréaliers français. I. - Variabilité entre années et entre départements du peuplement épi et du poids de grain par épi. Agronomie, 1983, 3 (9), pp.917-924. hal-00884590

\author{
HAL Id: hal-00884590 \\ https://hal.science/hal-00884590
}

Submitted on 1 Jan 1983

HAL is a multi-disciplinary open access archive for the deposit and dissemination of scientific research documents, whether they are published or not. The documents may come from teaching and research institutions in France or abroad, or from public or private research centers.
L'archive ouverte pluridisciplinaire HAL, est destinée au dépôt et à la diffusion de documents scientifiques de niveau recherche, publiés ou non, émanant des établissements d'enseignement et de recherche français ou étrangers, des laboratoires publics ou privés. 


\title{
Structure de la variabilité du rendement du blé d'hiver dans quelques départements céréaliers français. I. - Variabilité entre années et entre départements du peu- plement épi et du poids de grain par épi
}

\author{
Martine O. GUERIF
}

I.N.R.A., Station de Bioclimatologie, Centre de Recherches d'Avignon, BP 91, F84140 Montfavet

\begin{abstract}
RÉSUMÉ
A partir de résultats d'enquêtes sur la production du blé d'hiver portant sur 7 années dans 11 départements du Bassin parisien et de la région Centre, on se propose d'analyser la structure de la variabilité du rendement et de ses composantes en utilisant les deux critères Année et Région (la région est définie sur la base d'une différenciation entre systèmes culturaux). Il appararaît que (1) le système de culture permet un certain contrôle du peuplement épi alors que le poids de grain par épi est davantage soumis à la variabilité du climat ; (2) l'intensification du système de culture permet dans certains cas de s'affranchir de conditions climatiques défavorables.
\end{abstract}

Mots clés additionnels : Composantes du rendement, climat, système de culture.

Variability structure of winter wheat yield in some cereal-growing departments in France. I. Between-year and between-region variability of ear population and grain weight per ear.

Some results from field surveys of wheat production in 11 French Départements and 7 years have been used to analyse the structure of yield components variability, using the two criteria Year and Region (the region is defined on the basis of differentiation between cropping systems). Cropping system gave some control of ear population, while grain weight per car was more largely dependent on climatic variability. The intensification of the cultivation system sometimes allowed the effect of unfavourable climatic conditions to be minimized.

Additional key words : Yield components, climate, cropping system.

\section{INTRODUCTION}

La notion de composante du rendement est communément admise et considérée comme un outil essentiel dans l'étude de l'élaboration du rendement du blé. Les composantes sont en effet des entités élaborées pendant des périodes distinctes - sinon disjointes - du cycle végétatif ; elles ont des interrelations non négligeables liées aux phénomènes de compétition-compensation et sont diversement sensibles aux influences des techniques et du milieu. On arrive ainsi par leur intermédiaire à une meilleure compréhension du déterminisme du rendement (BALDY, 1973 ; BOIfFin et al., 1976 ; MASLE-MEYNARD, 1980).

Pour 11 départements du Bassin parisien et du Centre, nous disposons de 7 années de données de peuplement épi et de rendement sous forme de moyennes départementales (issues des Enquêtes Céréales réalisées par le SCEES*). Le. niveau (département) d'appréhension de ces données exclut toute tentative d'analyse déterministe de l'élaboration du rendement. Par contre, l'étude à cette échelle de la structure de leur variabilité spatiale et temporelle peut aider à dégager la ou les composantes qui limitent le rendement dans ces régions de culture et à définir ainsi les actions techniques prioritaires qui devraient être engagées. D'autre part, la période couverte par ces données (1971-78) est antérieure au développement récent du modèle de culture intensive (c'est-à-dire faisant appel à beaucoup d'« intrants») du blé, appuyé sur le terrain par des opérations de vulgarisation du type «Blé conseil»: une telle étude peut donc servir de référence (en tant qu'état initial) pour évaluer les modifications apportées par le développe-

(*) Service Central des Enquêtes et Etudes Statistiques du Ministère de l'Agriculture. 
ment de ces méthodes dans la variabilité du rendement. Par contre, la périocle étudiée est insuffisamment longue pour permettre de déceler une tendance à la hausse dans l'évolution des rendements sous l'effet des progrès réalisés dans les créations variétales et dans la technologie, et cela d'autant plus que la fin de la période est marquée par une suite de 3 années à faibles rendements (1975, 76 et 77 ).

\section{MATÉRIEL ET MÉTHODES}

L'enquête «Céréales» du SCEES est une enquête annuelle réalisée par sondage selon un plan d'échantillonnage en 2 phases (voir méthodologie, SCEES, 1976) : la $1^{\mathrm{re}}$, sur un échantillon d'exploitations, vise à l'estimation des superficies par espèce et par variété de céréale ; la $2^{\mathrm{e}}$, sur un échantillon de champs issu de la $1^{\text {re }}$ phase (en moyenne 80 champs par département), vise à l'estimation directe du peuplement épi et du rendement par prélèvement sur le terrain ( 2 placettes de $1 \mathrm{~m}^{2}$ par champ) : à cette occasion sont recueillis des renseignements sur les techniques culturales. Ce type de sondage ne permet pas d'obtenir des observations représentatives au niveau du champ : il est par contre tout à fait adapté à une étude qui se veut représentative à l'échelle régionale. Le nombre de départements soumis à l'enquéte est variable suivant les années: nous avons sélectionné les 11 départements du Centre et de l'Ile de France (fig. 1) qui ont subi l'enquête de façon continue de 1971 à 1978 (1974 manquant pour tous les départements, 1972 et 1973 pour le Loiret). Nous ne disposons pour

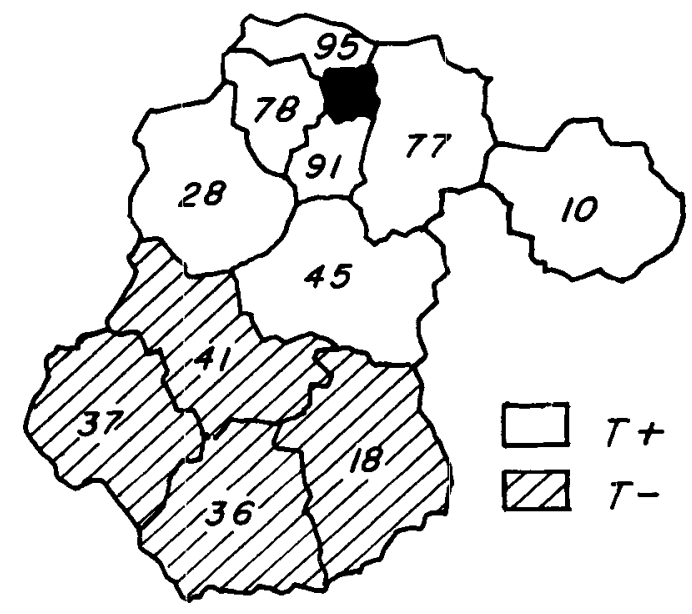

$\begin{array}{ll}10 \text { Aube } & 45 \text { Loiret } \\ 18 \text { Cher } & 77 \text { Seine-et-Marne } \\ 28 \text { Eure-et-Loir } & 78 \text { Yvelines } \\ 36 \text { Indre } & 91 \text { Essonne } \\ 37 \text { Indre-et-Loire } & 95 \text { Val d'Oise } \\ 41 \text { Loir-et-Cher } & \end{array}$

Figure 1

Les départements étudiés et les deux régions $T^{+}$et $T^{-}$. Départements studied and the two regions $T^{+}$and $T^{-}$.

l'instant que des données moyennes départementales et non pas au niveau du champ. Elles sont de 2 types :

a) un ensemble de données déclarées relatives aux variétés cultivées et aux principales techniques culturales mises en œuvre (précédent cultural ; type de préparation du sol ; date, densité et technique de semis ; fumures organique et minérale, nombre de désherbages...) disponibles sous forme de répartitions départementales: pour chaque don- née, les différentes modalités sont exprimées en p. 100 de la surface cultivée en blé ou du nombre de parcelles soumises à l'enquête.

b) des mesures objectives du rendement unitaire et du nombre d'épis par $\mathrm{m}^{2}$ (sauf pour le département 91, année 1978), sous forme de moyennes départementales. On peut opérer une décomposition du rendement en termes de peuplement épi et productivité de l'épi (poids de grain par épi $=$ rendement/nombre d'épis).

A cause de l'échelle à laquelle elles sont recueillies, il n'est pas envisageable de mettre directement en relation les deux types de données. Nous essaierons dans un premier temps de voir si et comment les distributions des pratiques culturales s'organisent en systèmes régionaux différenciés. Dans un deuxième temps, nous chercherons à établir le rôle de cette différenciation régionale dans la variabilité du rendement et de ses composantes. De la même façon, nous essaierons d'établir le rôle de l'année dans cette variabilité.

\section{RESULTATS}

\section{A. Régionalisation des systèmes de culture}

Nous avons réalisé une analyse factorielle des correspondances portant sur un tableau où :

- les colonnes sont formées par les distributions (en p. 100 de la superficie en blé qui a subi l'enquête dans le département) des modalités des différentes variables « techniques $»$. Dans un premier temps, nous avons proposé : variété, précédent cultural, technique de travail du sol, fumure organique et minérale, nombre de désherbages (le nombre de variables analysées est limité par les capacités du programme) ;

- les lignes sont formées par les départements $\times$ années.

L'examen de la projection des lignes et des colonnes suivant les axes factoriels 1 et 2 (représentée sur 2 graphes distincts pour plus de clarté) (fig. 2a et b) fait apparaître 2 groupes de départements correspondant à 2 types d'association des variables techniques, que l'on décrit par les caractéristiques qui les discriminent le plus :

- le groupe $\mathrm{T}^{+}$des départements du Bassin parisien (28, $95,78,91,77,10,45)$ où les précédents culturaux du blé sont, outre le blé lui-même, essentiellement des plantes sarclées (maïs, betterave à sucre, pomme de terre): on y ręncontre la pratique du non labour (NL), des désherbages multiples (D3), des engrais verts (03) et autres fumures organiques $(05)$, et les variétés récentes et productives.

- le groupe $\mathrm{T}^{-}$des départements du Centre $(41,18,36$, 37) où les précédents culturaux du blé sont, outre le blé, d'autres céréales (orge, avoine); d'autres plantes sarclées (fourragères), des cultures industrielles (oléagineux dont surtout colza) et fourragères: on y pratique le labour traditionnel (L) et les variétés cultivées sont plus anciennes.

Le $3^{\mathrm{e}}$ axe factoriel qui oppose les fumures minérales (surtout $\mathrm{P}$ et $\mathrm{K}$ ) fortes aux faibles induit une différenciation entre départements dans ces groupes :

- dans $\mathrm{T}^{+}$, les départements 10,28 et 91 pratiquent des fumures plus fortes que $78,45,77$, le département 95 pratiquant les fumures les plus faibles.

- dàns $\mathrm{T}^{-}$, le département 41 pratique des fumures plus fortes que $18,36,37$.

Dans un $2^{e}$ temps, nous avons remplacé les variables N P K par les variables date de semis et mode de semis; 
$\nabla$ Variétés (17)

- précédents culturaux

ja : jachère

ma : mais

bs : betterave à sucre

$p d t$ : pomme de terre

$\Delta s:$ autres plantes sarclées

$c i$ : cultures industrielles (oléagineux)

$b l$ : blé

$\Delta c:$ autres céréales

pa : prairie artificielle

$p n$ : prairie naturelle

$f a$ : fourrage annuel

$L$ labour NL non labour

\section{fertilisation $N P K$}

n 1 à 8

p 1 à 8

k 1 à 8
- fumure organique

2 fumier

3 engrais vert 1 pas de fumure

nombre de désherbages

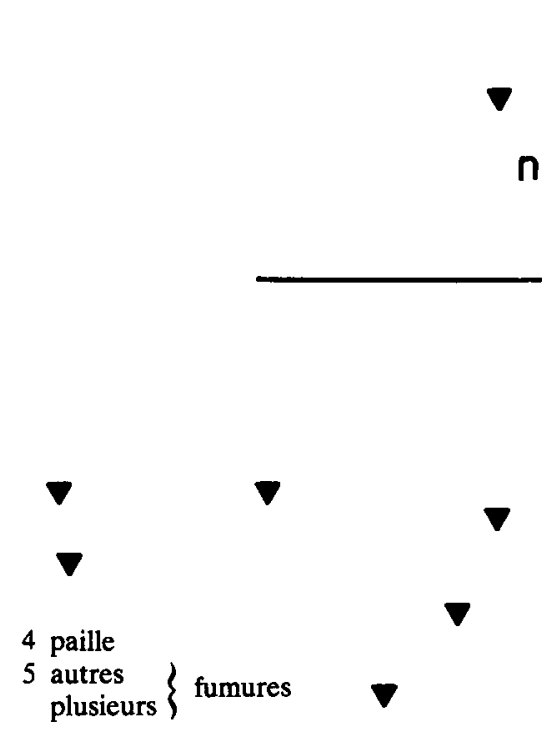

$0,1,2$

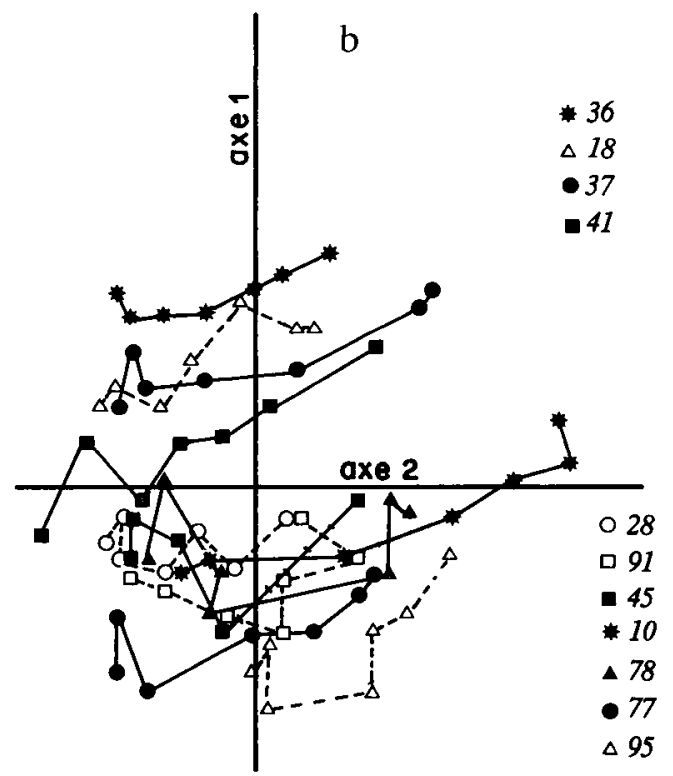

Figure 2

Analyse des correspondances - plan factoriel (1,2) (35 p. 100 de variance expliquée).

a) Projection des colonnes (variables).

b) Projection des lignes (individus).

Correspondance analysis. Factorial plan $(1,2)$ (35\% of variance explained).

a) Projection of rows.

b) Projection of columns. a

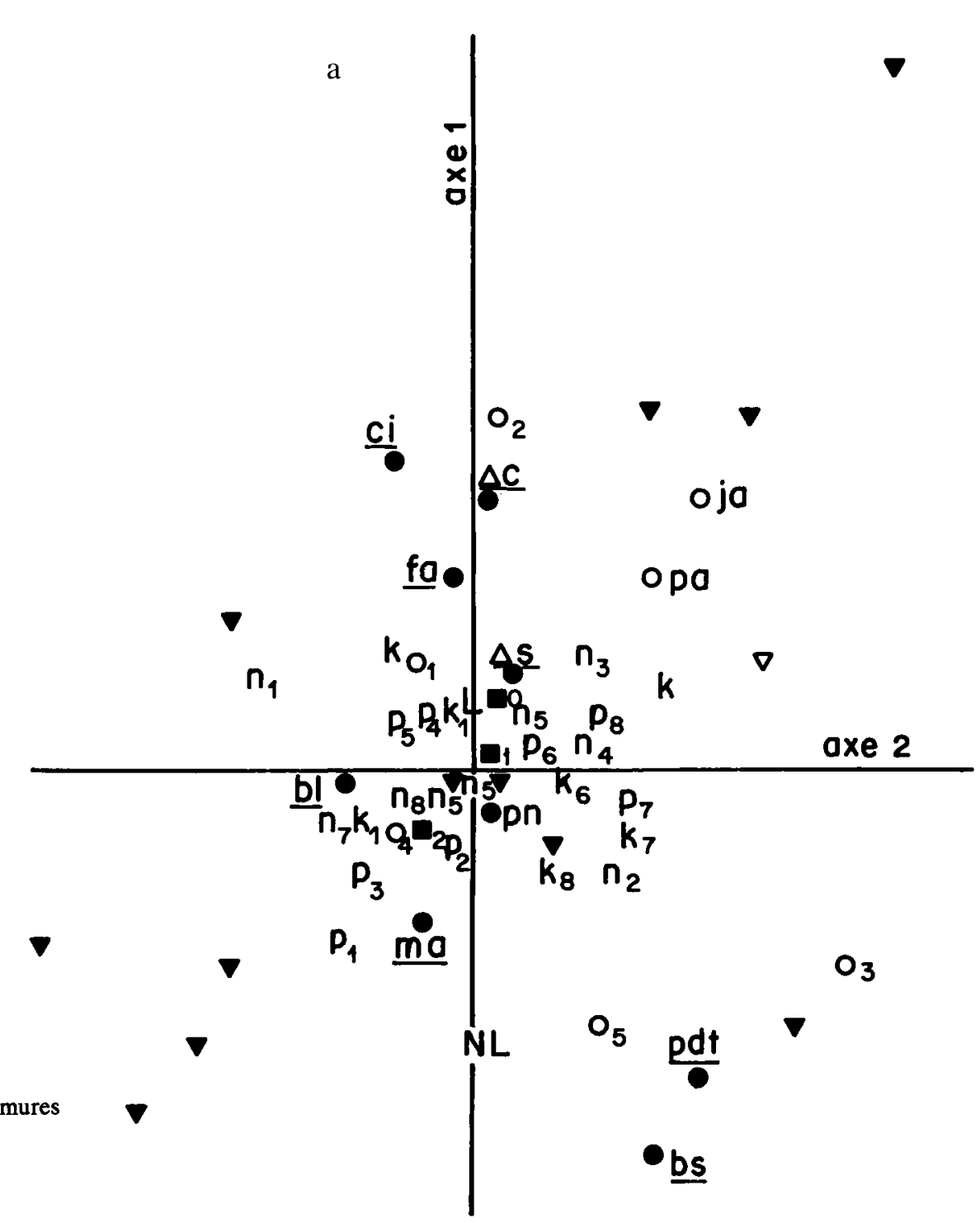

l'analyse confirme l'existence des 2 groupes, en précisant que :

- dans $\mathrm{T}^{+}$, on rencontre la pratique du semis à la volée et des dates de semis tardives (décembre, janvier) associées à l'absence de labour.

- dans $\mathrm{T}^{-}$, le labour est associé au semis en ligne traditionnel et les dates de semis sont plus précoces (octobre, novembre).

Ces 2 types d'association des variables techniques caractérisent 2 grands types de systèmes culturaux (le système cultural se définissant par une succession de cultures - approchée ici par les précédents culturaux rencontrés - et par les techniques culturales associées). On remarquera qu'ils correspondent à 2 régions formées de départements contigus.

\section{B. Etude de la variabilité du rendement et de ses composantes}

\section{Variabilité globale}

Sur l'ensemble des répétitions disponibles (75), le calcul des coefficients de variation montre que le rendement est l'élément le plus variable $(16,8$ p. 100$)$, suivi du poids de grain par épi $\left(13,3\right.$ p. 100) et que le nombre d'épis par $\mathrm{m}^{2}$ est la composante la plus stable $(10,9$ p. 100$)$. 


\section{Rôles des factours Années et Région dans la variabilité}

Pour les mettre en évidence, nous avons réalisé une analyse de la variance à 2 facteurs (Année: 7 niveaux, Régions: 2 niveaux) où les départements sont considérés comme des répétitions, ce qui revient à tester sur les 3 variables (Nombre d'épis, Poids de grain et Rendement) le modèle

$$
\chi_{i j k}==m+a_{i}+b_{j}+(a b) i j+\varepsilon i j k
$$

où $m$ est la moyenne générale

ai et $b_{j}$ les effets principaux (liés aux facteurs annćc et région)

$(\mathrm{ab})_{\text {if }}$ l'effet d'interaction entre les 2 facteurs

eijk sont les variables aléatoires résiduelles.

Les 2 effets principaux (tabl. 1) sont significatifs $(P \leqslant 0,001$ ou 0,01$)$ sur les 3 variables. L'interaction ne l'est $(P \leqslant 0,05)$ que pour le poids de grain et le rendement. Il est tout à fait remarquable que l'importance relative des 2 effets soit différente suivant la composante du rendement considérée : l'effet Région prédomine dans la variabilité du nombre d'épis alors que c'est l'effet Année qui prédomine pour le poids de grain. Globalement, sur le rendement, c'est l'effet Région qui prédomine.

\section{Structure de la variabilité}

La mise en correspondance des niveaux atteints par les composantes du rendement (sur un graphique, Nombre d'épis par $\mathrm{m}^{2} /$ Poids de grain par épi) avec les niveaux pris par les facteurs Région et Année permet d'interpréter le sens des effets de ces facteurs.

De manière à rendre la lecture plus aisée, 2 types de graphiques sont proposés: sur l'un, les individus sont représentés par leur localisation géographique (appartenance à un département ou une région); sur l'autre les mêmes individus sont représentés par le numéro de l'année. Dans le plan de chaque graphique on peut tracer un nouveau système d'axes tel qu'il est figuré sur la figure 3 :

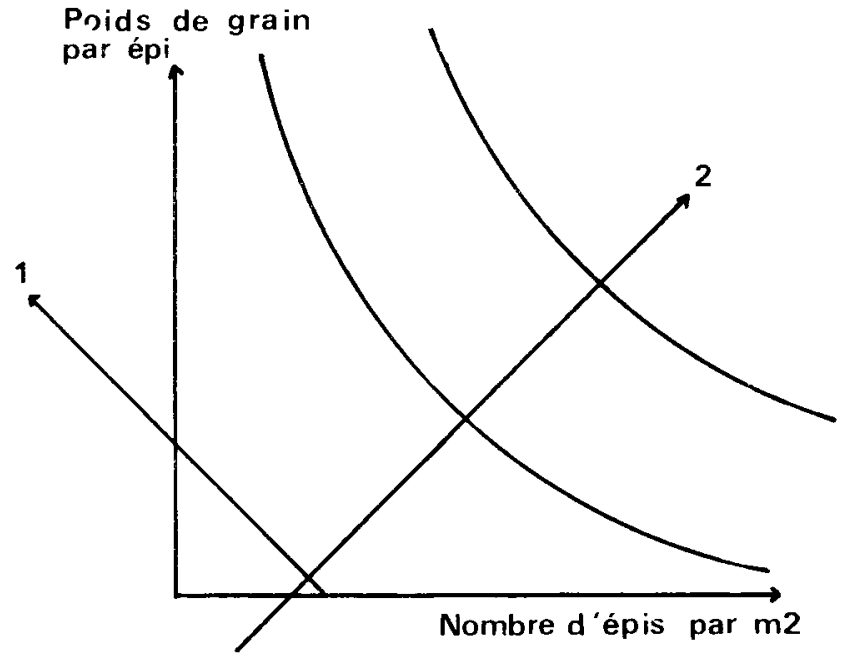

Figure 3

Les axes Nombre d'épis par $m^{2}$ et Poids de grain par épi et le nouveau système d'axes.

(1) Relation négative entre composantes.

(2) Relation positive entre composantes (rendement).

Axes Number of ears per $m^{2}$ and Grain weight per ear and the new set of axes.

(1) negative relation between yield components.

(2) positive relation between yield components $(=$ yield $)$.

l'un (1) représente la relation négative (compensation) qui existe entre les 2 composantes, l'autre (2) représente la relation positive : c'est l'axe des rendements croissants. Les hyperboles figurent des lignes de rendement constant.

Sur la figure 4 , où est portée la totalité des 75 points année $\times$ département, 2 effets apparaissent nettement :

a) les 2 régions $\mathrm{T}^{+}$et $\mathrm{T}^{-}$se discriminent (fig 4a) :

- par le niveau de rendement : les points de $\mathrm{T}^{-}$sont en majorité vers les faibles rendements, ceux de $\mathrm{T}^{+}$vers les forts.

\section{TABLEAU 1}

Tableau d'analyse de la variance. Les différences significatives sont marquées respectivement $\left(^{*}\right),\left({ }^{* *}\right),(* * *)$, pour $P \leqslant 0,05, P \leqslant 0,01$ et $P \leqslant 0.001$

Table of analysis of variance. Signijicant differences are indicated by $\left(^{*}\right),\left({ }^{* *}\right),\left({ }^{* *}\right)$, for $P \leqslant 0.05, P \leqslant 0.01$ and $P \leqslant 0.001$.

\begin{tabular}{|c|c|c|c|c|c|}
\hline & $\begin{array}{l}\text { Sources de } \\
\text { variations }\end{array}$ & DDL & $\begin{array}{c}\text { Somme des carrés } \\
\text { des écarts }\end{array}$ & $\begin{array}{l}\text { Carrés } \\
\text { moyens }\end{array}$ & $\mathrm{F}$ \\
\hline \multirow[t]{2}{*}{$\begin{array}{l}\text { Nombre } \\
\text { d'épis } \\
\text { par } m^{2}\end{array}$} & $\begin{array}{c}\text { Région } \\
\text { Année } \\
\text { Interaction } \\
\text { Var. résiduelle }\end{array}$ & $\begin{array}{r}1 \\
6 \\
6 \\
61\end{array}$ & $\begin{array}{r}20623 \\
46143 \\
9821 \\
97469\end{array}$ & $\begin{array}{r}20623 \\
7691 \\
1637 \\
1598\end{array}$ & $\begin{array}{c}12,91\left(^{* * *}\right) \\
4,81\left({ }^{* * *}\right) \\
1,02 \mathrm{NS}\end{array}$ \\
\hline & Total & 74 & 174056 & & \\
\hline \multirow[t]{2}{*}{$\begin{array}{c}\text { Poids } \\
\text { de grain } \\
\text { par épi } \\
(10 \mathrm{mg})\end{array}$} & $\begin{array}{c}\text { Région } \\
\text { Année } \\
\text { Interaction } \\
\text { Var. résiduelle }\end{array}$ & $\begin{array}{r}1 \\
6 \\
6 \\
61\end{array}$ & $\begin{array}{r}842 \\
10552 \\
1519 \\
5262\end{array}$ & $\begin{array}{r}842 \\
1759 \\
253 \\
86\end{array}$ & $\begin{array}{c}9,76\left(^{* *}\right) \\
20,39\left(^{* * *}\right) \\
2,94\left(^{*}\right)\end{array}$ \\
\hline & Total & & 18175 & & \\
\hline \multirow[t]{2}{*}{$\begin{array}{l}\text { Rendement } \\
(10 \mathrm{~kg} / \mathrm{ha})\end{array}$} & $\begin{array}{c}\text { Région } \\
\text { Année } \\
\text { Interaction } \\
\text { Var. résiduelle }\end{array}$ & $\begin{array}{r}1 \\
6 \\
6 \\
62\end{array}$ & $\begin{array}{r}76266 \\
304519 \\
31938 \\
140500\end{array}$ & $\begin{array}{r}76266 \\
50753 \\
5223 \\
2266\end{array}$ & $\begin{array}{c}33,66\left(^{* * *}\right) \\
22,40\left(^{* * *}\right) \\
2,30\left(^{*}\right)\end{array}$ \\
\hline & Total & 75 & 552623 & & \\
\hline
\end{tabular}



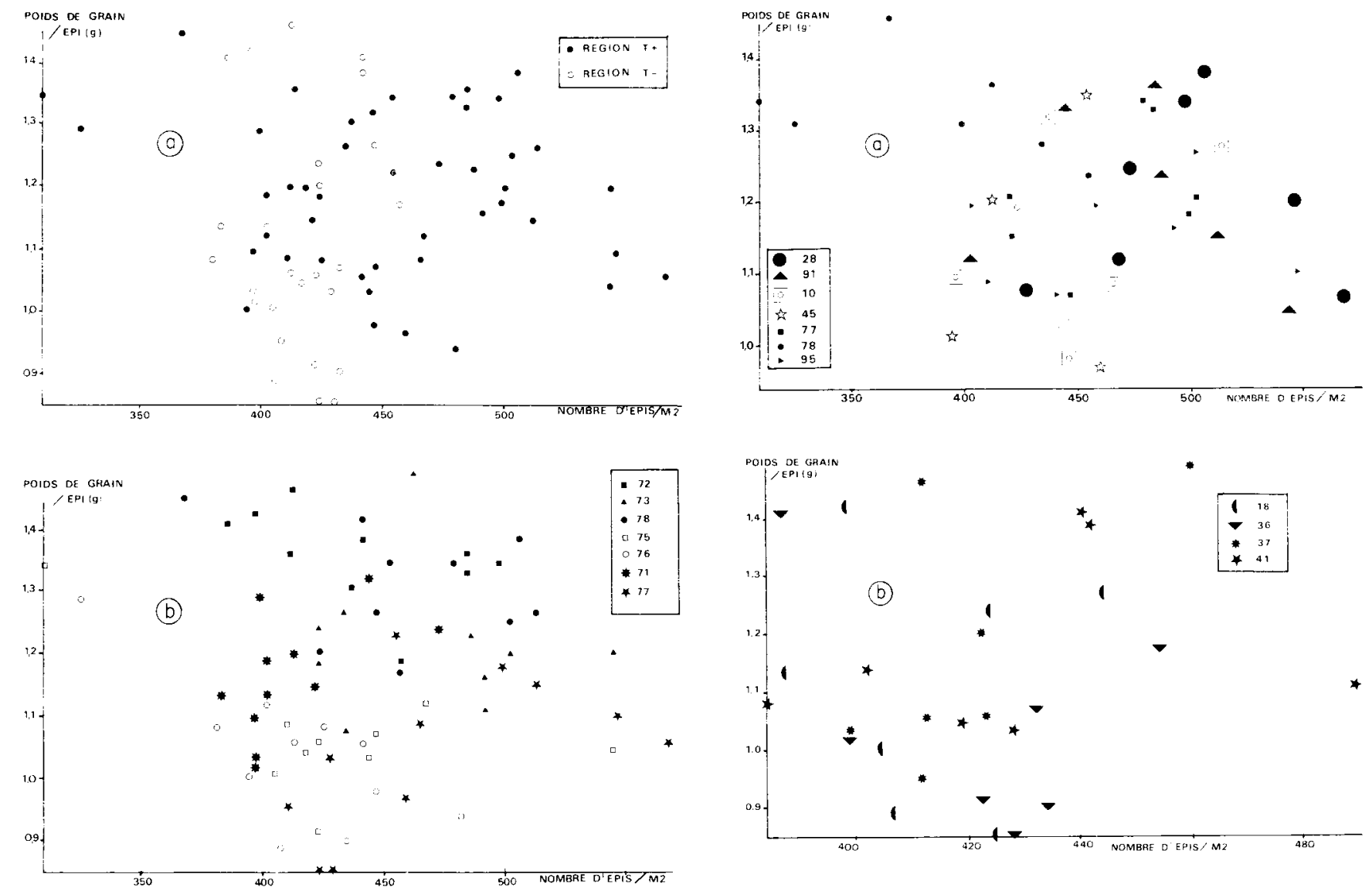

Figure 4

Projection des points département $\times$ année sur le plan défini par les axes Nombre d'épis par $m^{2}$ et Poids de grain par épi.

a) Les points sont repérés par leur appartenance à une région.

b) Les points sont repérés par l'année.

Projection of Departement $\times$ year points on the plane defined by the axes Number of ears per $m^{2}$ and Grain weight per ear.

Points are noted by:

a) The region they belong to.

b) The year.

- par le nombre d'épis : pour $\mathrm{T}^{-}$, les $\mathrm{N}$ épis restent dans une gamme étroite de valeurs faibles à moyennes; pour $\mathrm{T}^{+}$, la gamme est plus large et comprend, pour la moitié des cas, des valeurs élevées $(>450)$.

Il n'y a pas d'effet de la région sur le poids de grain pour lequel la gamme de variation est sensiblement identique pour $\mathrm{T}^{+}$et $\mathrm{T}^{-}$.

- par les modalités d'obtention d'un niveau de rendement donné : ainsi les forts rendements sont obtenus plutôt grâce à des forts nombres d'épis dans $\mathrm{T}^{+}$et grâce à de forts poids de grain par épi dans $\mathrm{T}^{-}$.

b) il existe un effet Année (fig. 4b) très marqué qui s'exprime par l'existence d'années «bonnes » $(1972,1973$, $1978)$ et « mauvaises» $(1975,1976)$ pour tous les départements, à la fois pour le rendement et pour les deux composantes. Par contre, les années 1971 et 1977 sont, selon les départements, bonnes ou mauvaises. L'effet Année s'exprime aussi sur les combinaisons entre composantes: plutôt N épi faible/P grain fort en 1971 et 1972, plutôt $\mathbf{N}$ épi fort/P grain faible en 1973 et 1977.

Si l'effet Région explique une grande part de la variabilité spatiale des rendements, il subsiste à l'intérieur de chacune des 2 régions une variabilité que l'on peut tenter de renseigner sur des graphes $\mathrm{N}$ épis/ $\mathrm{P}$ grain régionaux par l'appartenance à un département (fig. $5 \mathrm{a}$ et b).
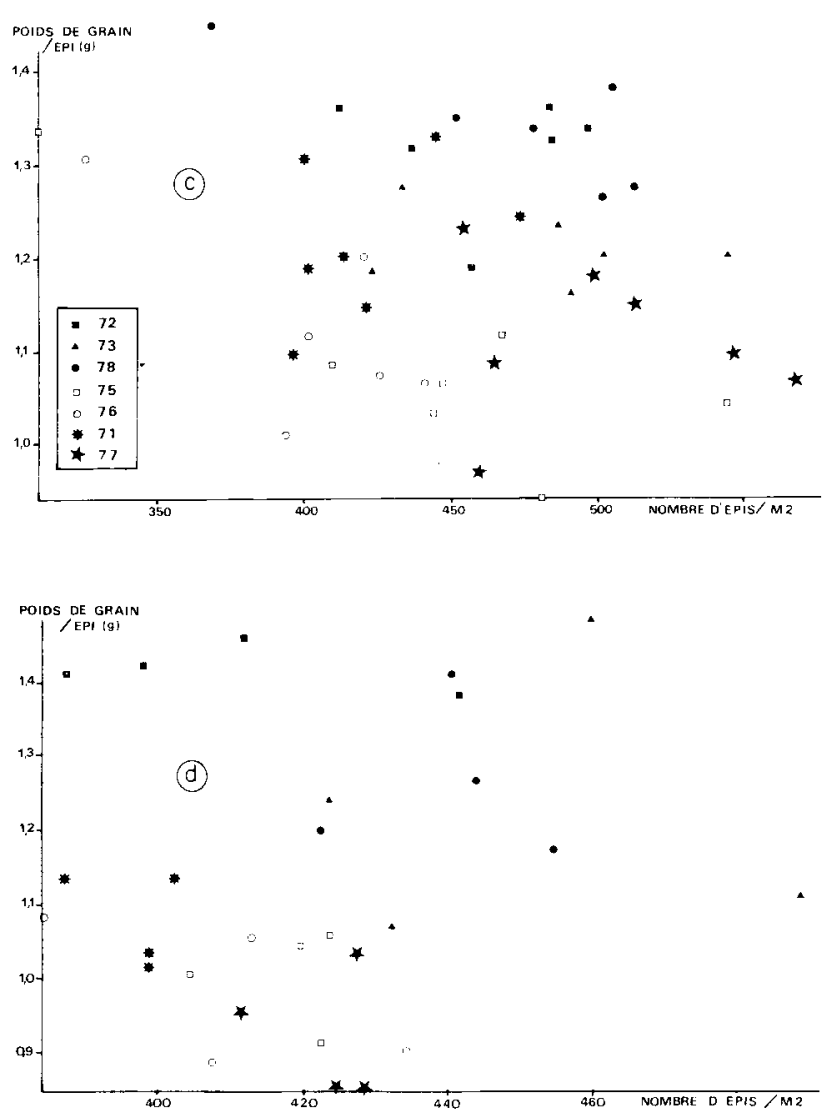

Figure 5

Projection par région des points département $\times$ année sur le plan défini par les axes Nombre d'épis par $m^{2}$ et Poids de grain par épi. a) et c) Région $T^{+}$.

b) et d) Région $T^{-}$

Projection, for each region, of Departement $\times$ year on the plane defined by the axes Number of ears per $m^{2}$ and Grãin weight per ear. 
On retrouve alors au sein de la région $\mathrm{T}^{+}$le même type de différence entre les départements 28 et 91 , d'une part, et 45 et 10 , d'autre part, qu'entre les 2 régions: les premiers réalisent en moyenne de meilleurs rendements grâce à de meilleurs nombres d'épis. Le département 78 se singularise par des rendernents en moyenne plus faibles dus à des nombres d'épis par $\mathrm{m}^{2}$ toujours inféricurs à 450 dont l'effet sur le rendement est plus ou moins compensé par un poids de grain par épi toujours élevé (entre 1,2 et $1,5 \mathrm{~g}$ ). La région $\mathrm{T}^{+}$apparaît donc plus hétérogène de ce point de vue que la région $\mathrm{T}^{-}$.

Sur les graphes régionaux où les individus sont représentés par l'année (fig. $5 \mathrm{c}$ et $\mathrm{d}$ ), on retrouve les bonnes et les mauvaises années. Mais les années 1971 et 1977 sont assimilées dans la région $\mathrm{T}^{-}$au groupe des mauvaises années; dans la région $\mathrm{T}^{+}$au contraire, la plupart de ces points-années (particulièrement les départements 28,91 et l'année 1977) passent du côté des bons rendements, grâce à un fort nombre d'épis dans le cas de 1977, à un fort poids de grain dans le cas de 1971.

Le cas de ces années 1971 et 1977 illustre parfaitement l'interaction région $\times$ année (et même dans $\mathrm{T}^{+}$, l'interaction département $\times$ année).

\section{DISCUSSION ET CONCLUSION}

On peut ainsi résumer les résultats obtenus :

1. la variabilité du nombre d'épis est liée de façon prépondérante à un effet spatial (régional ou départemental) ;

2. la variabilité du poids de grain est liée de façon prépondérante à un effet interannuel;

3. l'effet interannuel s'exprime par une action à la fois sur le niveau des composantes du rendement (années «bonnes » ou « mauvaises ") et sur le type de combinaison des composantes permettant d'obtenir un niveau de rendement donné ;

4. il y a une différence de comportement entre les 2 régions (et entre départements pour $\mathrm{T}^{+}$)

- dans les valeurs de nombre d'épis et de rendement moyens (supérieures pour $\mathrm{T}^{+}$et, à l'intérieur de $\mathrm{T}^{+}$, pour les départements 28 et 91 );

- dans la combinaison des composantes pour atteindre de bons rendements (par le nombre d'épis pour $\mathrm{T}^{+}$, par le poids de grain pour $\mathrm{T}^{-}$) ;

5. l'interaction lieu $\times$ année mise en évidence dans l'analyse de variance s'exprime particulièrement bien pour les années intermédiaires 1971 et 1977 , où les résultats sont très différents suivant que l'on est dans $\mathrm{T}^{+}$ou dans $\mathrm{T}^{-}$. Cette interaction joue essentiellement sur le poids de grain par épi et par conséquent le rendement.

Ces résultats sur la structure de la variabilité du rendement et de ses composantes ont un caractère uniquement descriptif. Leur interprétation passe par la traduction des 2 composantes de la variabilité en termes plus explicites. Le niveau d'apprétension des données recueillies ne permet que d'avancer des hypothèses.

Pour la composante spatiale : on a vu que la variabilité du rendement et de ses composantes était en rapport avec les régions $\mathrm{T}^{+}$et $\mathrm{T}^{--}$. Or, étant donné la cohérence géographique de ces 2 régions, la différenciation que l'on observe entre leurs comportements respectifs recouvre sans doute des disparités d'ordre climatique et pédologique. Mais il est également très probable qu'il y a un effet important des différences (mises en évidence de façon très schématique au II, a) entre les systèmes de culture pratiqués dans ces régions. Le système de culture intègre en fait en partie les caractéristiques pédoclimatiques de la région. Il existe en effet une adaptation des cultures et des techniques aux conditions du milieu: c'est ce que constate SEBILLOTTE (1975) dans son analyse de l'évolution des systèmes de culture depuis 1950.

Pour la composante interannuelle, 2 types de facteurs interviennent : les facteurs climatiques et l'évolution dans le temps des variétés cultivées et des techniques culturales. Pour ces dernières, on peut citer :

- l'évolution des précédents culturaux, marquée par la diminution à partir de 1975-1976 des précécents maïs (ex. : pour l'Eure-et-Loir, ils passent de 63 p. 100 des cas en 1973 à 39 p. 100 en 1978)

- le développement au cours des années 1970 de l'application des fongicides qui n'a malheureusement pas été noté au cours de ces enquêtes.

Le sens global de l'effet de ces évolutions n'est pas facilement décelable. La répartition aléatoire des bonnes et mauvaises années semble démontrer que le climat a un rôle primordial dans la variabilité interannuelle des composantes du rendement. On sait en outre que les mauvaises années sont dues à des accidents climatiques (excès d'eau à l'automne et au printemps en 1975, sécheresse en 1976) et qu'une bonne année comme 1978 est caractérisée dans cette région par des conditions climatiques très favorables pendant le tallage, la montaison et le remplissage des grains (ITCF, 1976, 1978).

Ainsi, peut-on, avec quelques réserves, associer variabilité spatiale à effet du système de culture et variabilité interannuelle à effet du climat. Ainsi le système de culture a un rôle prépondérant dans la variabilité du nombre d'épis (on retrouve là des résultats analytiques sur le rôle des techniques - densité, date de semis, nutrition azotée - sur le nombre d'épis), alors que le poids de grain reste davantage influencé par le climat. La finalité des techniques culturales est bien sûr de permettre dans un milieu donné l'expression des potentiels variétaux en maîtrisant aux mieux les interactions milieu $\times$ peuplement végétal, de façon à s'affranchir le plus possible des aléas climatiques. Les résultats obtenus ici montrent que :

1) par leur niveau de technicité, en effet, certains départements parviennent à obtenir de très bons rendements les bonnes années grâce à une meilleure efficacité au niveau du peuplement épi. Il faut noter cependant qu'ils ne parviennent pas dans ces cas là à réaliser de très bons poids de grain par épi (sans doute à cause de phénomènes de compétition entre épis): il semble que les techniques culturales pratiquées ne sont pas de nature à lever cette limite à l'obtention de rendements supérieurs; on retrouve là la moindre influence des techniques culturales dans la variabilité du poids de grain.

2) Outre cet effet sur la meilleure expression des rendements potentiels, on a vu un autre effet de l'action des techniques culturales : le contrôle de la dépendance peuplement végétal/climat, c'est-à-dire un certain affranchissement vis-à-vis de conditions climatiques défavorables. Cet effet se manifeste clairement en 1977 où l'interaction région $x$ année est typiquement une interaction technique $\times$ climat : en effet, selon les observations de l'ITCF (1977), le développement important de maladies favorisé par les conditions climatiques a provoqué un échaudage parasitaire - donc des poids de grain faibles (cf. fig. 5c et 
d) - limité en présence de traitements fongicides, vraisemblablement plus fréquisils dans la région $\mathrm{T}^{+}$. On vérifie ici le rôle important joué par une technique culturale - l'application de fongicides - dans la suppression d'une limite d'origine climatique, et on ne peut que regretter l'absence de notation sur cette technique dans l'enquête "Céréales".

3) Cette maîtrise des interactions milieu $\times$ peuplement végétal, qui passe par l'utilisation d'une quantité croissante d' « intrants» (quantité de semence, fertilisation, traitements phytosanitaires...) n'apparait cependant pas suffisante sur la période considérée puisque la variabilité interannuelle du rendement reste élevéc et qu'on n'arrive pas à corriger l'effet de conditions climatiques très défavorables (1975, 1976).

En cela, nos résultats constituent un témoin intéressant pour apprécier si les techniques de «blés intensifs » récem- ment développées atteignent l'objectif de réduire la variabilité interannuelle des rendements.

Sur le plan de l'analyse, ces résultats doivent être approfondis de façon à mettre en évidence le poids des différents facteurs évoqués - dont le facteur variétal - C'est ce que nous permettra de faire l'exploitation des données de l'enquête "Céréales » au niveau élémentaire de la parcelle dont nous présenterons les résultats dans un second mémoire.

Reçu le 16 avril 1982. Accepté le 25 mai 1983.

\section{REMERCIEMENTS}

Je remercie le S.C.E.E.S. et particulièrement Monsieur B. Pous, du Centre de Toulouse, qui m'ont permis et facilité l'accès aux résultats des Enquêtes Céréales.

\section{RÉFÉRENCES BIBLIOGRAPHIQUES}

Baldy C., 1973. Etude de peuplements de blé tendre (Triticum aestivum $L$. em. Thell.): Croissance, répartition de la lumiere et assimilation nette. Thèse Doct. Ing., Université Paris-Sud, Orsay, 27 avril 1973, 109 p. + annexes.

Boiffin F., Sebillotte M., Couvreur F., 1976. Incidence de la simplification du travail du sol sur l'élaboration des rendements du blé et du maïs. Colloque ITCF «Simplification du travail du sol en production céréalière », 7-8 décembre 1976.

Masle-Meynard J., 1980. L'élaboration du nombre d'épis chez le blé d'hiver. Influence de différentes caractéristiques de la structure du peuplement sur l'utilisation de l'azote et de la lumière. Thèse de Doct. Ing., INAPG, 24 janvier 1980, 274 p.
S.C.E.E.S., 1976. Résultats des enquêtes sur les productions céréalières en 1975, p. 126-135, Collections de Statistique Agricole, étude $\mathrm{n}^{\circ} 146$.

I.T.C.F. (a), 1976. Conditions climatiques de la campagne 1975 1976.

I.T.C.F. (b), 1977. Conditions climatiques de la campagne 19761977 et conséquences sur le développement du blé tendre d'hiver. I.T.C.F. (c), 1978. Conditions climatiques de la campagne 1977 1978 et conséquences sur le développement du blé tendre d'hiver. Notes internes SEA - Secteur Physiologie ITCF.

Sebillotte M., 1975. Évolution des systèmes de culture et des moyens de production. Journ. natle. d'inf. "Techniques nouvelles de production du blé », I.T.C.F., Paris, p. 25-70. 